\section{Salud mental de hombres mayores en Chile: una realidad por priorizar}

\author{
José M. Aravena, ${ }^{1}$ Jean Gajardo, ${ }^{2} y$ \\ Rodrigo Saguez ${ }^{3}$
}

Forma de citar Aravena JM, Gajardo J, Saguez R. Salud mental de hombres mayores en Chile: una realidad por priorizar. Rev Panam Salud Publica. 2018;42:e121. https://doi.org/10.26633/ RPSP.2018.121

\section{RESUMEN}

Los problemas de salud mental constituyen un conjunto de condiciones de alto impacto. Las personas de 60 y más años presentan vulnerabilidades particulares que aumentan el riesgo de experimentar problemas de salud mental y consecuencias terminales como el suicidio. En este grupo etario, los hombres constituyen un subgrupo poco considerado como destinatarios particulares de acciones en salud. En el caso de Chile, este artículo busca caracterizar la realidad de la salud mental de los hombres adultos mayores mediante cifras provenientes de encuestas poblacionales realizadas en el país (Encuesta Nacional de Salud [ENS] 20032009, Estudio Nacional de Dependencia en las Personas Mayores [ENADEAM] 2009, Encuesta de Caracterización Socioeconómica Nacional [CASEN] 2015). El artículo aborda indicadores relativos a depresión y sintomas depresivos, suicidio e ideación suicida, y consultas de salud mental. Las cifras reportadas en las encuestas seleccionadas informan que, en Chile, los hombres adultos mayores son el grupo que presenta la mayor tasa de suicidio, el menor número de ideación e intento suicida, y la menor frecuencia reportada de consultas de salud mental. De igual modo, presentan

\footnotetext{
Facultad de Salud, Universidad Bernardo O'Higgins, Santiago, Chile.

2 Facultad de Medicina, Universidad San Sebastián, Santiago, Chile. Enviar correspondencia a Jean Gajardo, jean.gajardo@gmail.com

Unidad de Nutrición Pública, Instituto de Nutrición y Tecnología de los Alimentos, Universidad de Chile, Santiago, Chile.
}

menor reporte de depresión en comparación con mujeres. Estas cifras permiten aproximar a un perfil de salud mental en hombres mayores en Chile y cuestionar la pertinencia de los actuales modelos epidemiológicos para la identificación de perfiles de riesgo de salud mental en este grupo. Asimismo, emerge la urgente necesidad de diseñar programas de salud que incorporen dicha consideración de género, para lograr una adecuada pesquisa y aceptabilidad de las potenciales intervenciones dirigidas a promover la salud mental y reducir riesgos en hombres adultos mayores.

Palabras clave Suicidio; adulto mayor; salud mental; salud del hombre; Chile.

Los hombres adultos mayores son la población que reporta menor sintomatología depresiva e ideación suicida a nivel mundial (1). Sin embargo, constituye la población que presenta la tasa de suicidio más alta por 100000 habitantes, en especial después de los 70 años de edad (2). A su vez, los hombres mayores tienen una baja utilización de servicios de salud de atención primaria y, sobre todo, servicios de salud mental (3). Con base en dichos antecedentes, resulta pertinente observar la situación de los hombres adultos mayores en el contexto latinoamericano.

Chile es uno de los países con mayor número de personas con depresión de la Organización para el Desarrollo y Cooperación Económicos (OECD) y supera el promedio mundial $(4,4 \%$ ) con 5\% (844 253 personas mayores de 15 años) (1). Estas cifras se enmarcan en un país en transición demográfica avanzada; en el CENSO 2017 se estimó que 16,2\% de la población tenía 65 años y más de edad (44,3\% hombres; $55,7 \%$ mujeres) (4). En este sentido, se establece la relevancia de analizar la situación de salud mental de las personas mayores.

El presente artículo tiene como objetivo describir las características de salud mental de los hombres adultos mayores en Chile, específicamente en el reporte de síntomas depresivos, suicidio, ideación suicida y uso de servicios de salud, mediante el análisis de las diferencias existentes según género y grupos etarios. Estas cifras han sido extraídas de las siguientes encuestas poblacionales llevadas a cabo en Chile: Encuesta Nacional de Salud (ENS) 2003 y 2009 (6, 7), Estudio Nacional de Dependencia en las Personas Mayores (ENADEAM) 2009 (8), Encuesta de Caracterización Socioeconómica Nacional (CASEN) 2015 (4), datos provenientes del Departamento de 
Estadística e Información en Salud (DEIS) 2013 (9) e información del Estudio Chileno de Prevalencia Psiquiátrica (ECPP) 2002 (10). La información incluida en los estudios ENS, ENADEAM y ECPP se extrae en forma directa de su fuente de publicación. Para los datos extraídos del DEIS, se calcularon las tasas de suicidio en personas mayores de 60 años por cada 100000 sujetos. Por último, de la encuesta CASEN se extrajeron datos referentes al número de consultas de salud mental en el último año y se calcularon proporciones para diseñar tabla con diferencias entre grupos etarios y género.

A partir de esta información, se espera contribuir al reconocimiento de las características de salud mental de los hombres mayores en Chile, contrastar diferencias de género y de edad y otorgar recomendaciones para una mejor detección de problemas y riesgos de salud mental.

\section{Reporte de síntomas depresivos}

Para indagar en la frecuencia de depresión de adultos mayores en Chile, se dispone de la ENS (6), versiones 2003 y 2009. En esta encuesta fue utilizada la Entrevista de Diagnóstico Internacional Compuesta en forma abreviada (CIDI-SF) para establecer la presencia de episodio depresivo mayor en los últimos 12 meses. La ENS 2003 incluyó un total de 3616 personas y, en la muestra, $11,0 \%$ fueron personas de 65 años y más (918 personas). La encuesta estimó una prevalencia de depresión de 16,8\% (intervalo de confianza de 95\% [IC95\%]: 12,720,9) en personas mayores (7). Por su parte, la ENS 2009 refirió una disminución en la prevalencia de cuadros depresivos reportados en esta población, estimando que el 11, 2\% (IC95\%: 8,1-15,1) de las personas mayores entrevistadas (901 personas) presentaron depresión según CIDI-SF, con 16,9\% (IC95\%: 12-23,2) en mujeres y 4,1\% (IC95\%: 2-8,2) en el caso de los hombres (7).

No obstante, existe una discrepancia entre estas cifras y las reportadas en el ENADEAM (8). En este estudio, que consideró una muestra representativa nacional de personas mayores (4 766 personas), se utilizó el instrumento Escala de Depresión Geriátrica de 15 ítems (GDS-15) para establecer la presencia de depresión durante las últimas dos semanas. El estudio estimó que $18 \%$ de los sujetos incluidos presentó depresión, que es el problema de salud más prevalente luego de la hipertensión $(62,1 \%)$ y la diabetes $(21,8 \%)(8)$.

\section{Suicidio en los hombres mayores}

El suicidio es un antecedente de gran relevancia sobre la salud mental en las personas mayores. Tanto en Chile como en el mundo, el grupo etario que presenta las mayores tasas de suicidio son las personas mayores de 80 años $(2,11)$. En países como Estados Unidos se reporta una muerte por cada 100 o 200 intentos entre las personas de 15 a 24 años de edad, mientras que entre las personas mayores de 65 años se reporta un suicidio por cada cuatro intentos (11).
En el caso de Chile, de acuerdo con datos provenientes del DEIS durante 2013, las mayores tasas de suicidio por cada 100000 habitantes fueron reportadas en el grupo etario de 70 años y más, y alcanza su máxima ocurrencia en las personas de 80 y más años. En este grupo, son los hombres mayores los que aportan de forma más significativa: los sujetos de 70-74 años presentaron tasas por cada 100000 habitantes de 30,0\%, y los de 80 años y más, tasas cercanas a 40,0\%, en comparación con las mujeres de esta misma edad que presentaron tasas inferiores a 5,0\% (9). De este modo, los hombres de 70 y más años presentaron las tasas más elevadas de suicidio en la población total.

Considerando que los intentos suicidas y la ideación suicida suelen utilizarse como predictores de un intento suicida futuro, Silva y colaboradores (10) realizaron, en el año 2013, un estudio en una muestra aleatoria de cuatro provincias de Chile, con el propósito de conocer la prevalencia de ideación e intentos suicidas en adultos de 15 y más años. Dicho estudio refirió que las personas de 65 y más años son la población que reportó menor prevalencia de intentos suicidas $(2,3 \%)$, ideación suicida $(12,2 \%)$ y deseo de estar muerto $(21,4 \%)$. No obstante, fue el grupo etario que reportó mayor pensamiento sobre la muerte $(38,9 \%)(10)$. Si se consideran los primeros indicadores para efectos de estimación del riesgo individual, podría pensarse que los hombres mayores tienen un menor riesgo de cometer suicidio. No obstante, dicha hipótesis se desecha al contrastar con la tasa de suicidios cometidos.

\section{Utilización de servicios de salud mental}

En personas adultas mayores con depresión, la subutilización de servicios de salud mental se asocia con un bajo tratamiento de condiciones de salud mental e incrementa el riesgo suicida y mortalidad no suicida (12). En Estados Unidos, las personas mayores de 65 años con depresión reportan menos visitas a servicios de salud mental y presentan una menor probabilidad de consultar con otros profesionales que no sean su médico habitual (13).

En relación con la situación en Chile, datos encontrados en la Encuesta CASEN 2015 (14), estudio constituido de una muestra representativa nacional con sobremuestreo de 87420 personas residentes en 324 comunas, muestran que $21,06 \%$ de las personas de 60 años y más presentan al menos una consulta de salud mental en el último año. Del total de estas consultas, $75,6 \%$ corresponde a mujeres (14).

Asimismo, 23,43\% de las mujeres de 60 años y más presentan al menos una consulta de salud mental, en contraste con $16,04 \%$ de los hombres del mismo grupo etario. En este caso, los hombres de 60 años y más presentan la menor proporción y frecuencia de consultas de salud mental junto a las mujeres de 0 a 15 años de edad (10,62\% en mujeres de 0 a 15 años) (14).

En relación con las consultas de salud mental en mayores de 60 años, del total de sujetos de 60 a 69 años de edad, 2,66\% presenta al menos una consulta de salud mental en el último año (3,82\% de mujeres y $1,29 \%$ de 
FIGURA 1. Porcentaje de sujetos que realizaron al menos una consulta de salud mental durante 2015, según grupo etario

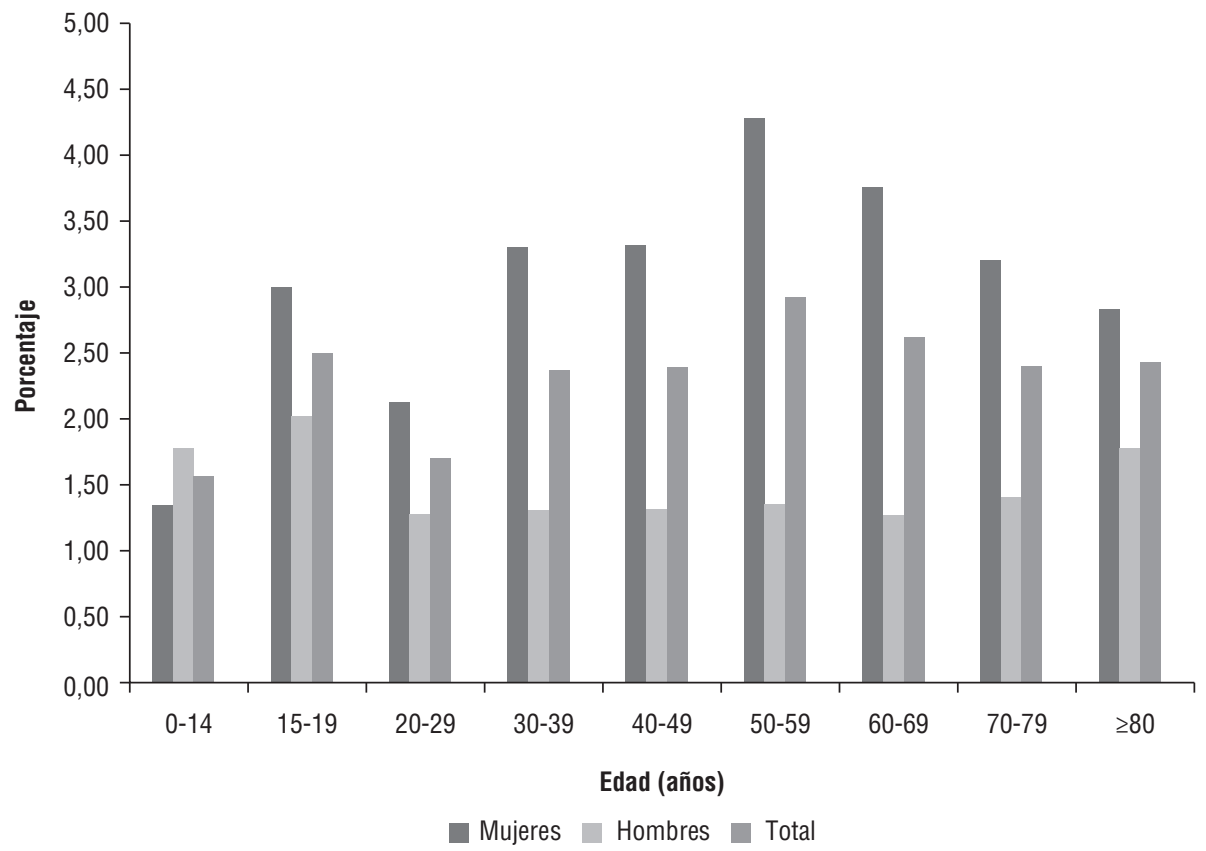

Elaborada por los autores a partir de datos publicados y de acceso público de la encuesta CASEN 2015 (14).

hombres). Por su parte, en los sujetos de 70 a 79 años, $2,44 \%$ (3,26\% de mujeres y 1,43\% de hombres) presentó al menos una visita. Por último, en personas de 80 años y más, $2,47 \%$ (2,88\% de mujeres y $1,81 \%$ de hombres) presentaron al menos una consulta de salud mental (14) (figura 1).

\section{DISCUSIÓN}

En este artículo se presentan antecedentes extraídos de encuestas poblacionales que permiten identificar que los hombres mayores en Chile son el grupo etario que reporta menor sintomatología depresiva, ideación suicida e intentos suicidas, aunque a la vez constituye el grupo que presenta la mayor tasa de suicidio (en especial después de los 70 años). De igual modo, junto con las niñas de hasta 15 años de edad, los hombres adultos mayores son el grupo etario que reporta menor frecuencia de consultas de salud mental y, de modo general, reportan menor cantidad de consultas que las mujeres de su misma edad.

Los trastornos depresivos tienen menores prevalencias estimadas en hombres que en mujeres mayores. Sin embargo, existe evidencia que señala que la depresión en las personas mayores puede acompañarse de síntomas diferenciados (15), sumado a que los hombres pueden presentar con mayor frecuencia síntomas como irritabilidad, estrés o apatía, en vez de tristeza. Sería importante cuestionar, por una parte, si la expresión de esta enfermedad en hombres adultos mayores presenta características clínicas que las pruebas de tamizaje utilizadas hoy en día en Chile pueden no ser capaces de captar. Por otro lado, aunque los hombres mayores reporten menor sintomatología depresiva, son el grupo que presenta mayor tasa de suicidios en comparación con la población total, pero que al mismo tiempo reportan menor prevalencia de intentos suicidas e ideación suicida, habituales indicadores centrales de riesgo suicida a nivel individual. A partir de este hecho, y considerando que son uno de los grupos que reporta menor acceso a servicios de salud mental, la detección individual de casos de hombres mayores con problemas de salud mental en servicios de atención primaria debería ser una prioridad y un signo de alerta para seguimiento por un elevado riesgo suicida, si se tiene en cuenta que la depresión, el trastorno bipolar y la comorbilidad son los factores que se asocian de mayor forma con suicidios cometidos en hombres mayores (16). El diagnóstico poco certero puede llevar a la prescripción inapropiada de psicofármacos en adultos mayores (sobre todo de benzodiacepinas), en especial en hombres y sujetos sin diagnósticos de salud mental (17). Lo anterior no corresponde a una responsabilidad propia y exclusiva de los equipos de salud, que deben prepararse con mayor profundidad en la atención de las personas mayores y su salud mental, sino que también refleja la necesidad de un sistema que sea capaz de identificar y responder de forma rápida y eficiente a estos sujetos.

En este escenario, la probabilidad de rescate es baja en comparación con otros grupos, lo que desafía los mecanismos actuales de detección de estas situaciones y cómo la epidemiología local ha construido el concepto riesgo suicida y los factores involucrados, más bien desde un foco en la población general y sin observar las potenciales diferencias en subgrupos, resal- 
tando de mayor forma el riesgo en población adolescente mediante campañas preventivas del suicidio. Antecedentes locales señalan que, en Chile, los hombres adultos mayores tienen una menor participación en los programas de promoción y prevención disponibles en atención primaria en salud. En este punto, resulta pertinente que los sistemas de salud evalúen sus estrategias de vinculación con este grupo específico y apunten a mecanismos de pesquisa y apoyo con mayor aceptabilidad y efectividad, con visibilización de las necesidades de personas que pueden transformarse con facilidad en grupos ocultos.

\section{CONCLUSIÓN}

Los hombres mayores en Chile son una población que desafía el abordaje actual de salud mental en el sistema de salud local, puesto que reportan baja sintomatología depresiva y se ven expuestos a subdiagnóstico de depresión. Asimismo, constituyen un grupo demográfico en alto riesgo, debido a que presentan la tasa de suicidio más alta de la población, pero el menor reporte de ideación e intentos suicidas, así como una baja utilización de servicios de salud mental.

Con base en estos hechos, se recomienda desarrollar e incrementar la sensibilidad del diagnóstico de salud mental en hombres, con la inclusión de métodos de tamizaje específicos para la población mayor y establecimiento de parámetros diferenciados de identificación de riesgo de depresión y suicidio, como la soledad y la comorbilidad. Debería aumentar la capacitación a los equipos de salud en la pesquisa de hombres mayores en riesgo y definir un abordaje pertinente según género y factores contextuales, con aumento de la investigación sobre barreras y facilitadores que influyen en el uso y adherencia de los servicios de salud mental y atención primaria.

Conflicto de intereses. Ninguno declarado por los autores.

Declaración. Las opiniones expresadas en este manuscrito son responsabilidad del autor y no reflejan necesariamente los criterios ni la política de la RPSP/ PAJPH y/o de la OPS.

\section{REFERENCIAS}

1. World Health Organization. Depression and other common mental disorders global health estimates, 2017. Disponible en: http:// apps.who.int/iris/bitstream/10665/ 254610/1/WHO-MSD-MER-2017.2eng.pdf?ua $=1$

2. World Health Organization (WHO). Preventing suicide: a global imperative. Geneva: WHO; 2014. Disponible en: http://apps.who.int/iris/bitstream/10665/131056/1/9789241564 779 eng.pdf

3. Simming $A$, van Wijngaarden $E$, Fisher SG, Richardson TM, Conwell Y. Mental health care need and service utilization in older adults. Am J Geriatr Psychiatry. 2012;20(5): 441-51.

4. Instituto Nacional de Estadísticas de Chile (INE). Síntesis de Resultados CENSO 2017. INE, 2018. Disponible en: http:/ / www.censo2017.cl/descargas/ home / sintesis-de-resultadoscenso2017.pdf

5. Thumala D, Kennedy BK, Calvo E, Gonzalez-Billault C, Zitko P, Lillo P, et al. Aging and health policies in Chile: new agendas for research. Health Syst Reform. 2017;3(4):253-60. Disponible en: https://www. tandfonline.com/doi/full/10.1080/ 23288604.2017.1353844

6. Ministerio de Salud de Chile (MINSAL). Encuesta Nacional de
Salud 2009-2010. Santiago de Chile: MINSAL; 2010.

7. Markkula N, Zitko P, Peña $S$, Margozzini P, Retamal C. P. Prevalence, trends, correlates and treatment of depression in Chile in 2003 to 2010. Soc Psychiatry Psychiatr Epidemiol. 2017;52(4):399-409.

8. Albala C, Sánchez R H, Fuentes G A, Lera M L, Cea N X. Estudio nacional de la dependencia en las personas mayores en Chile. Santiago de Chile; 2010. Disponible en: http://www. senama.cl/filesapp / Estudio_ dependencia.pdf

9. Ministerio de Salud de Chile. Departamento de Estadística e Información en Salud. Indicadores básicos de salud, 2017. Disponible en: http://www.deis.cl/indicadoresbasicos-de-salud/

10. Silva D, Vicente B, Saldivia S, Kohn R. Conducta suicida y trastornos psiquiátricos en Chile, un estudio poblacional. Rev Med Chil. 2013;141(10): 1275-82.

11. Jahn DR. Suicide risk in older adults: the role and responsibility of primary care. JCOM. 2017;24(4):181-92.

12. National Alliance on Mental Illness (NAMI). Depression in older persons-Fact sheet. Arlington: NAMI; 2009. Disponible en: http://www2. nami.org/Content/Navigation Menu/Mental_Illnesses /
Depression / Depression_Older Persons_FactSheet_2009.pdf

13. Crabb R, Hunsley J. Utilization of mental health care services among older adults with depression. J Clin Psychol. 2006;62(3):299-312.

14. Ministerio de Desarrollo Social de Chile. CASEN 2015 Bases de datos. Observatorio Social, 2015. Disponible en: http://observatorio. ministeriodesarrollosocial.gob.cl/ casen-multidimensional/casen/ basedatos.php

15. Warren T. Depression in the elderly. N Engl J Med. 2014;371:1228-36.

16. Yi SW. Depressive symptoms on the geriatric depression scale and suicide deaths in older middle-aged men: a prospective cohort study. J Prev Med Public Heal. 2016;49(3):176-82.

17. Maust DT, Blow FC, Wiechers IR, Kales HC, Marcus SC. National trends in antidepressant, benzodiazepine, and other sedative-hypnotic treatment of older adults in psychiatric and primary care. J Clin Psychiatry. 2017;78(4):e363-71.

Manuscrito recibido el 19 de noviembre de 2017. Aceptado para su publicación, tras revisión, el 23 de mayo de 2018. 
ABSTRACT

\section{Mental health in older men in Chile: a reality to be prioritized}

Keywords
Mental health problems are a set of high-impact conditions. People aged 60 years and over are particularly vulnerable to factors that increase their risk of experiencing mental health problems and fatal outcomes, such as suicide. Within this age group, men as a subgroup are seldom the targets of relevant public health measures. This article aims to characterize the mental health status of men aged 60 years and over in Chile by using data from population-based surveys carried out in the country: the 2003 and 2009 National Health Surveys (NHS), the 2009 National Study of Dependency in Older Persons (ENADEAM), and the 2015 National Socioeconomic Characterization Survey (CASEN). The article looks at indicators for depression and depressive symptoms, suicide, and suicidal ideation, as well as mental health visits. According to reported figures from these surveys, older men in Chile constitute the population subgroup with the highest suicide rate, the lowest reported rates of suicidal ideation and suicide attempts, and the lowest reported frequency of mental health visits. Furthermore, men report depression less often than women. These figures provide an approximate picture of the mental health profile of older men in Chile and give rise to questions regarding the relevance of current epidemiological models for the identification of mental health risk profiles in this group. They also point to the urgent need to design health programs that integrate gender considerations in order to ensure proper screening and the acceptability of potential interventions for promoting mental health and reducing risk factors among older men.

Suicide; aged; mental health; men's health; Chile.
RESUMO

\section{Saúde mental de homens idosos no Chile: uma realidade a ser priorizada}

Os problemas de saúde mental constituem um conjunto de enfermidades com grande repercussão. Os indivíduos de 60 anos ou acima têm vulnerabilidades específicas que aumentam o risco de apresentar problemas de saúde mental com consequências fatais, como o suicídio. O sexo masculino é um subgrupo desta faixa etária pouco considerado como alvo em particular de ações em saúde. Este artigo enfoca o caso do Chile e busca caracterizar a realidade da saúde mental dos homens idosos a partir de dados obtidos em pesquisas de base populacional realizadas no país: Enquete Nacional de Saúde (ENS) 2003-2009, Estudo Nacional de Dependência nos Idosos (ENADEAM) 2009 e Pesquisa de Caracterização Socioeconômica Nacional (CAS) 2015. São apresentados os indicadores relativos a depressão e sintomas depressivos, suicídio e ideação suicida e consultas de saúde mental. Os dados informados nas pesquisas selecionadas revelam que, no país, os homens idosos apresentam a taxa de suicídio mais alta, o menor número de casos de ideação suicida e tentativa de suicídio e a menor frequência referida de consultas de saúde mental. Igualmente, eles referem menos depressão que as mulheres. Estes dados permitem traçar um perfil da saúde mental em homens idosos no Chile e questionar a adequação dos modelos epidemiológicos atuais para identificar os perfis de risco de saúde mental neste grupo. Existe também uma necessidade premente de estabelecer programas de saúde que incorporem o fator gênero para que se possa realizar uma pesquisa adequada com aceitabilidade das possíveis intervenções para promover a saúde mental e reduzir os riscos em homens idosos.

Suicídio; idoso; saúde mental; saúde do homem; Chile. 